\title{
Predictive utility of neutrophil-to-lymphocyte ratio and platelet-to-lymphocyte ratio in intraductal papillary neoplasm of the bile duct
}

Vor Luvira', Supot Kamsa-ard², Ake Pugkhem', Varisara Luvira', Tharatip Srisuk1, Attapol Titapun', Artit Silsirivanit', Sopit Wongkham ${ }^{1}$, Narong Khuntikeo' ${ }^{1}$, Chawalit Pairojkul', Vajarabhongsa Bhudhisawasdi'

'Faculty of Medicine, Khon Kaen University, Thailand

2Faculty of Public Health, Khon Kaen University, Thailand

\begin{abstract}
Aim of the study: Intraductal papillary neoplasm of the bile duct (IPNB) can present at various stages of the disease. Each stage needs different treatment. The neutrophil-to-lymphocyte ratio (NLR) and the platelet-to-lymphocyte ratio (PLR) have been described as predictive markers for several tumors. There has been no investigation on the role of NLR and PLR in IPNB.

Material and methods: We retrospectively reviewed the medical records of 112 patients who underwent curative-intent hepatic resection for IPNB between January 2007 and December 2011. All clinical parameters and survival were analyzed for their association with NLR and PLR.

Results: For prediction of malignancy, the best respective cut-off for NLR and PLR was 2.74 and 130, with area under the ROC curve being 0.662 and 0.763 . For micro-papillary IPNB, both markers well predict malignancy and lymph node involvement. The respective area under the ROC curve of NLR and PLR for prediction of malignancy was 0.78 and 0.88 . Both markers had an area under the ROC curve for prediction of lymph node involvement of 1.0. The median overall survival of those with $P L R<130$ was 86.4 months compared with 45.0 months for those with PLR > $130(p=0.02)$.

Conclusions: NLR and PLR seem likely candidates for predicting malignancy, lymph node involvement, and survival of the patients. PLR performed better than NLR for all predictions. The markers worked very well for micro-papillary IPNB; however, we recommend using these markers in conjunction with the radiologic appearance of tumors.

Key words: intraductal papillary neoplasm of the bile duct, neutrophil-to-lymphocyte ratio, platelet-to-lymphocyte ratio, predictive marker.
\end{abstract}

Address for correspondence

Dr. Vor Luvira, Faculty of Medicine, Khon Kaen University, Thailand, e-mail: vor_110@yahoo.com

\section{Introduction}

Intraductal papillary neoplasm of the bile duct (IPNB) is considered a distinct bile duct tumor, with a wide spectrum of disease from benign to malignant [1]. At presentation, patients can present with various stages of disease, needing different treatments $[1,2]$. We classified IPNB according to its morphology on pre-operative imaging in order to predict the likelihood of malignancy and lymph node involvement. There, however, remains heterogeneity within the same IPNB morphology [2] - hence the critical need for predictive markers that can assist in planning IPNB surgery.

The neutrophil-to-lymphocyte ratio (NLR) and the platelet-to-lymphocyte ratio (PLR) are inflammatory markers [3] which have been correlated with poor survival in many malignancies [4-7]. Both markers, moreover, have been verified as predictive of intraductal papillary mucinous neoplasms (IPMN-P) $[8,9]$, which is considered the pancreatic counterpart of IPNB. There has, however, been no study on the role of NLR and PLR for predicting the malignancy of IPNB. 
To achieve a better understanding of the utility of NLR and PLR as predictive markers in IPNB, we investigated the association between the likelihood of malignancy and lymph node involvement in IPNB as a guide to the treatment of IPNB.

\section{Material and methods}

\section{Patient}

Patients undergoing hepatic resection for pathologically confirmed IPNB at Srinagarind Hospital, Khon Kaen University between January 2007 and December 2011 were identified from a prospectively maintained pathological database. In the current study, IPNB included both benign and malignant pathologically confirmed papillary tumors, within grossly dilated bile ducts, and the presence of a fibrovascular core upon microscopy. Accordingly, the intraductal growth type of intrahepatic cholangiocarcinoma and the papillary type of extrahepatic cholangiocarcinoma were included as malignant IPNB. We reviewed clinical and laboratory parameters including age, sex, liver function test, tumor markers, white blood cells and differential count, and preoperative imaging features.

In the study, we used our proposed classification of IPNB to define the utility of NLR and PLR according to the morphology of the IPNB [2]. We categorized IPNB into 5 classes: class I - classical intrahepatic IPNB (presence of an intraductal tumor with unilateral intrahepatic duct dilatation); class II - extrahepatic IPNB (presence of an intraductal tumor with bilateral intrahepatic duct dilatation); class III - cystic variant (cystic tumor with a papillary tumor inside and the presence of bile duct communication); class IV - micropapillary lesion (disproportional bile duct dilatation in the absence of any discernible tumor); and class $\mathrm{V}$ - macroinvasion (presence of a mass-forming tumor incorporated with intraductal tumor) [2].

Resectability was evaluated using cross-sectional imaging before surgery (i.e., computed tomography and magnetic resonance imaging). All surgical operations were planned to achieve gross tumor removal as a minimum. All surgical specimens were sent to the Department of Pathology for diagnosis and staging.

\section{Neutrophil-to-lymphocyte ratio and the platelet-to-lymphocyte ratio}

All white blood cell and differential counts were taken on the day prior to surgery. None of the patients had any signs of infection at the time the specimen(s) were taken. NLR was calculated as the ratio of the neutrophil counts divided by the lymphocyte counts and the PLR was the ratio of platelet counts divided by the lymphocyte counts.

\section{Ethical consideration}

The Institutional Review Board (IRB), Office of Human Research Ethics, Khon Kaen University, reviewed and approved the present study (HE591513).

\section{Statistical analysis}

Continuous variables were reported as means $( \pm$ SDs), medians (minima : maxima). Categorical variables were reported as a whole number and percentage. Survival analysis was performed using the Kaplan-Meier analysis. Patients who died from other causes or had peri-operative death (defined as death within 30 days after surgery) were censored from the analysis. Comparisons among groups were analyzed using a log-rank test. Calculation of NLR and PLR was performed by plotting the respective receiver operating characteristic curve in order to assess the capacity of the index to predict the invasiveness of IPNB and lymph node involvement. A $p$-value of $<0.05$ was considered statistically significant. All statistical analyses were performed using STATA version 13. (Lakeway, TX, USA).

\section{Results}

\section{Demographic data}

A total of 112 patients underwent liver resection for pathologically confirmed IPNB. There were 75 men $(67 \%)$ and 37 women (33\%), but there was no difference in the sex distribution between benign and malignant IPNB. The mean age at diagnosis was 59.3 years $(\mathrm{SD}=$ 8.8). The mean age of benign IPNB was slightly higher than that in the malignant group (62.1 vs. 59.0). According to our proposed classification, most of the IPNBs in the study were class I (intrahepatic intraductal IPNB). There was no benign IPNB in class II (extrahepatic IPNB) or class V (macro-invasive IPNB). In order to normalize serum bilirubin before surgery, the patients with obstructive jaundice, only the patients with extrahepatic IPNB (class II), received preoperative biliary drainage. The respective median serum bilirubin level for IPNB class I, II, III, IV and V was 0.5, 1.9, 0.4, 0.4 and $0.4 \mathrm{mg} / \mathrm{dl}$. The comparison of clinical data between benign and malignant IPNB is summarized in Table 1.

\section{Utilities of neutrophil-to-lymphocyte ratio, the platelet-to-lymphocyte ratio}

The respective median NLR for benign and malignant IPNB was $2.03(1.08: 12.29)$ and $2.88(0.52: 22.5)$. 
Table 1. Clinico-pathologic characteristics according to level of invasiveness of IPNB (benign vs. malignant)

\begin{tabular}{|c|c|c|}
\hline $\begin{array}{l}\text { Clinico-pathologic } \\
\text { variables }\end{array}$ & $\begin{array}{l}\text { Benign } \\
(n=17)\end{array}$ & $\begin{array}{c}\text { Malignant } \\
(n=95)\end{array}$ \\
\hline \multicolumn{3}{|l|}{ Age (years) } \\
\hline$<60$ & $7(41.2 \%)$ & $51(53.7 \%)$ \\
\hline$\geqslant 60$ & $10(58.8 \%)$ & $44(46.3 \%)$ \\
\hline Mean \pm SD & $62.1 \pm 9.5$ & $59.0 \pm 8.8$ \\
\hline \multicolumn{3}{|l|}{ Sex } \\
\hline Male & $13(76.5 \%)$ & $62(65.3 \%)$ \\
\hline Female & $4(23.5 \%)$ & $33(34.7 \%)$ \\
\hline Liver enzymes & $n=17$ & $n=88$ \\
\hline \multicolumn{3}{|l|}{ Median (min : max) } \\
\hline AST (IU/I) & $32(17: 186)$ & $33(12: 303)$ \\
\hline ALT (IU/I) & $39(15: 345)$ & $32(4: 521)$ \\
\hline ALP (IU/I) & $92(53: 451)$ & $153(50: 817)$ \\
\hline CA 19-9 ( $\mu \mathrm{g} / \mathrm{ml})$ & $n=12$ & $n=76$ \\
\hline Median (min : max) & $32(0.6: 1000)$ & $29(0: 6510)$ \\
\hline \multicolumn{3}{|l|}{ Hepatectomy } \\
\hline Right/Extended right & $8(47.1 \%)$ & $52(54.7 \%)$ \\
\hline Left/Extended left & $9(52.9 \%)$ & $43(45.3 \%)$ \\
\hline \multicolumn{3}{|l|}{ LN dissection } \\
\hline Yes & $11(64.7 \%)$ & 77 (81.1\%) \\
\hline No & $6(35.3 \%)$ & 18 (18.9\%) \\
\hline \multicolumn{3}{|l|}{ Class of IPNB } \\
\hline Class I & $8(47.0 \%)$ & $42(44.2 \%)$ \\
\hline Class II & $0(0.0 \%)$ & $12(12.6 \%)$ \\
\hline Class III & $2(11.8 \%)$ & $3(3.2 \%)$ \\
\hline Class IV & $7(41.2 \%)$ & $8(8.4 \%)$ \\
\hline Class V & $0(0.0 \%)$ & $30(31.6 \%)$ \\
\hline \multicolumn{3}{|l|}{ NLR } \\
\hline Median (min : max) & $2.03(1.08: 12.29)$ & $2.88(0.52: 22.5)$ \\
\hline \multicolumn{3}{|l|}{ PLR } \\
\hline Median (min : max) & $98.3(12.4: 330.7)$ & $157.0(21.1: 1027)$ \\
\hline
\end{tabular}



The respective median PLR for benign and malignant IPNB was 98.3 (12.4:330.7) and 157.0 (21.1: 1027). The distribution of NLR and PLR between the benign and malignant IPNB is presented in Figure 1. Further analysis revealed no statistically significant difference between NLR and PLR. The respective ability of NLR and PLR to predict malignant IPNB was assessed using the area under the receiver operator characteristic curve (Fig. 2). For NLR, the area under the ROC curve was 0.662 , with the best cut-off point of 2.74 (with a respective sensitivity and specificity of $54.7 \%$ and $82.4 \%$ ). The specificity increased to $100 \%$ using a cut-off point of 12.7. For PLR, the area under the ROC curve was 0.763 , with the best cut-off point of 130 (with a respective sensitivity and specificity of $67.4 \%$ and $88.2 \%$ ).

We further explored the respective utility of NLR and PLR for predicting malignancy and likelihood of lymph node metastasis of the IPNB as per the morphologic classification (Table 2). Amongst our group of patients extrahepatic IPNB (class II) and macroinvasive IPNB (class V) were all malignant, and there was no lymph node metastasis among patients with cystic IPNB (class III). As for micro-papillary IPNB (class IV), both NLR and PLR very well predicted the likelihood of malignancy and lymph node metastasis. For prediction of malignancy, the best cut-off for NLR was 1.53 , providing a respective sensitivity and specificity of $87.5 \%$ and $71.4 \%$ (area under ROC $=0.78$ ). By comparison, the best cut-off for PLR was 118.3, providing a respective sensitivity and specificity of $75 \%$ and $85.7 \%$ (area under ROC $=0.88$ ). For prediction of lymph node involvement, both NLR and PLR provided a sensitivity and specificity of $100 \%$ at a respective cutoff of 4.59 and 217 (area under ROC curve 1.0) (Fig. 3).

\section{Patient survival}

At the median follow up of 92.9 months, the respective 1-, 3-, and 5-year survival rate was $87.4 \%$

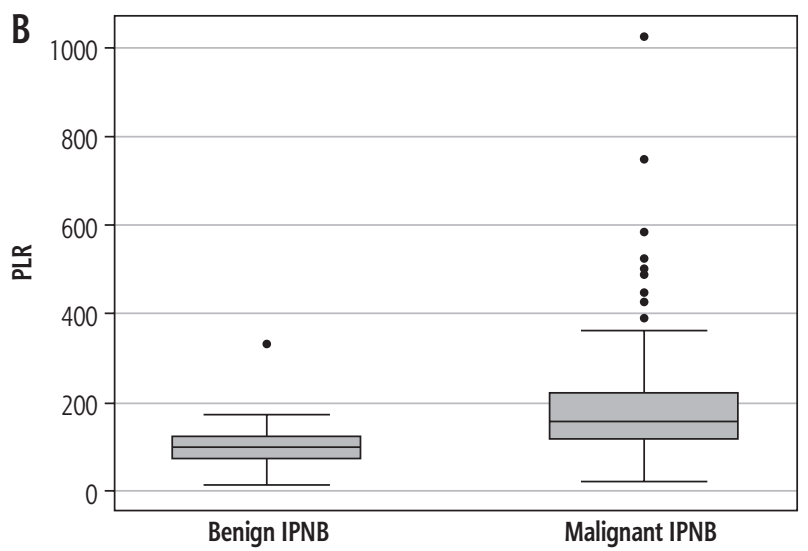

Fig. 1. Box plot of (A) NLR and (B) PLR distribution between benign and malignant IPNB 



Fig. 2. Receiver operating characteristics (ROC) curve for prediction of malignant IPNB of (A) NLR and (B) PLR

(95\% CI: 79.7-92.3), 65.8\% (95\% CI: 56.1-73.8), and $47.6 \%$ (95\% CI: 38.0-56.5). The median overall survival was 58.6 months (95\% CI: 43.9-74.5). A PLR of $>130$ predicted the poorest overall survival, such that the median overall survival of those with a PLR $<130$ was 86.4 months compared with 45.0 months for those with a PLR $>130(p=0.02)$. There was no association between NLR and overall survival (Fig. 4, $p=0.44$ ).

\section{Discussion}

The study established that NLR and PLR can be used as predictive markers for IPNB. PLR seems to be better than NLR for all predictions, including for malignancy, lymph node involvement, and patient survival. The ability of prediction was increased if these markers were used in conjunction with the radiological appearance of the tumor.

NLR and PLR are inflammatory markers known to be associated with invasive cancer in many tumors, especially IPMN $[8,9]$, which is considered the pancreatic counterpart of IPNB $[10,11]$ and both develop from the endoderm foregut almost at the same time. Interestingly, the lining epithelia of the bile duct and main pancreatic duct show similar morphologies and phenotypes, and both are accompanied by periductal glands. Furthermore, the exocrine pancreatic acini are remnantly found in the peribiliary glands. Based on these findings, it seems plausible that the biliary tract has features of pancreatic elements in addition to the duct system, which is specialized for the drainage of bile secreted by hepatic parenchyma, particularly, hepatocytes. Interestingly, some pancreatic and biliary diseases show similar pathological features and even biological behaviors. For example, extrahepatic cholangiocarcinoma and ductal adenocarcinoma of the pancreas share many clinicopathological features. Both
Table 2. Area under ROC curve for predicting malignant IPNB and lymph node involvement

\begin{tabular}{lcc}
\hline \multirow{2}{*}{ Class } & \multicolumn{2}{c}{ Area under curve of predictors } \\
\cline { 2 - 3 } & NLR & PLR \\
\hline Malignant IPNB & & \\
\hline 1 & 0.4777 & 0.6190 \\
\hline 2 & - & - \\
\hline 3 & 0.5000 & 0.5000 \\
\hline 4 & 0.7679 & 0.8750 \\
\hline 5 & - & - \\
\hline LN involvement & & \\
\hline 1 & 0.3417 & 0.4917 \\
\hline 2 & 0.5185 & 0.5926 \\
\hline 3 & - & - \\
\hline 4 & 1.0000 & 1.0000 \\
\hline 5 & 0.6184 & 0.5329 \\
\hline
\end{tabular}

of them are hypothesized to arise from similar preneoplastic and early neoplastic intraepithelial lesions. Intraductal papillary tumors, with frequent mucin hyperproduction, develop in the pancreas (intraductal papillary mucinous neoplasm. In the current study, PLR was found to be slightly more effective than NLR. Both NLR and PLR provided less predictive ability if they were used to predict all types of IPNB regardless of morphology. However, vis-à-vis micro-papillary IPNB (class IV in our classification), the predictive ability of both markers was high, possibly because the markers were increased from both the tumors and an intrinsic response to other causes (i.e., infection) [3]. As with tumors, those least likely to cause biliary obstruction, (e.g., micro-papillary lesion), they may gain benefit from using the inflammatory markers as predictive markers. NLR and PLR were also found to be less effective vis-à-vis extrahepatic cholangiocarcino- 

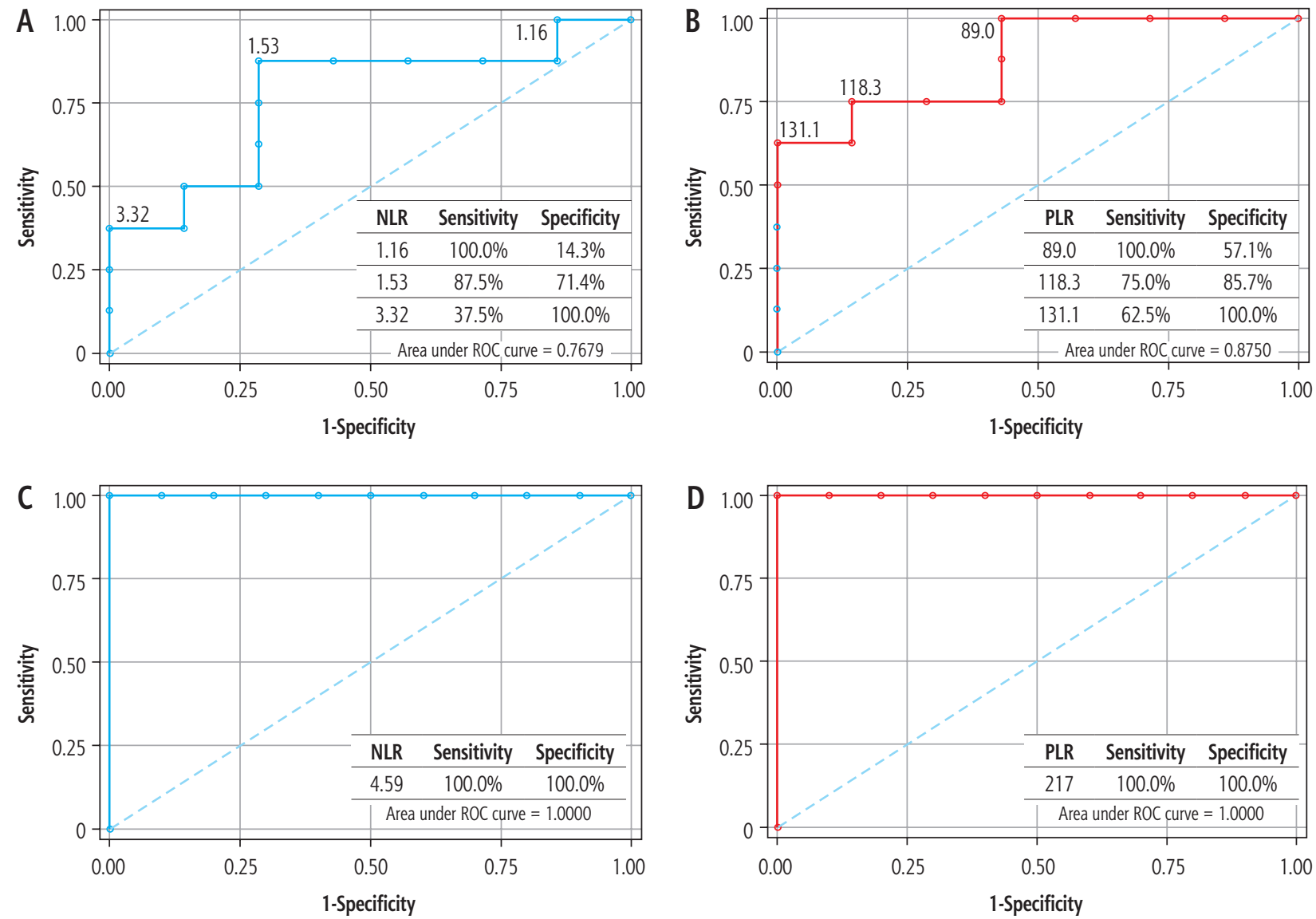

Fig. 3. Receiver operating characteristics (ROC) curve for prediction of micro-papillary IPNB. A, B) Prediction of malignant micro-papillary IPNB; A) NLR and (B) PLR. C, D) Prediction of lymph node involvement in micro-papillary IPNB, C) NLR and (D) PLR

ma compared with intrahepatic cholangiocarcinoma [7, 12-16]. Moreover, according to our classification, some classes (extrahepatic, IPNB with liver invasion) have a very high likelihood of malignancy. In cystic IPNB, there was no lymph node involvement [2], and the markers are less useful. We thus recommend that, before using these inflammatory markers, the likely



NLR $<2.74 \quad--$ NLR $>2.74$ tumor should be predetermined according to the radiologic appearance.

The role of routine lymph node dissection for IPNB is not established $[1,17]$. This slowly progressing tumor may derive some benefit from lymph node dissection [18]; however, based on a previous study, the survival benefit of routine lymph node dissection could not

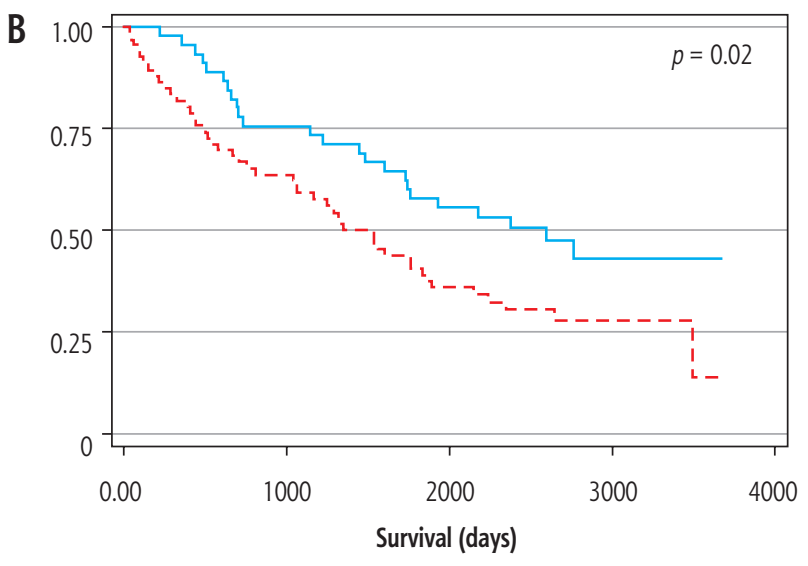

- PLR $<130 \quad---$ PLR $>130$

Fig. 4. Kaplan-Meier survival curve of IPNB patients treated by hepatic resection with respect to (A) NLR and (B) PLR 
be demonstrated, owing to the very low rate of lymph node involvement [1]. We performed further analysis and found that in IPNB with liver invasion (class V), the rate of lymph node involvement approached 30\% [2], and lymph node dissection yielded a survival benefit (unpublished). We had recommended that routine lymph node dissection be performed for extrahepatic IPNB and IPNB with liver invasion [2]. For other classes, if lymph node involvement could be predicted in IPNB, patients who would be suitable for lymph node dissection could be selected. Thus, using NLR and PLR, the dissection of regional lymph node is recommended if NLR is $>22.5$ or PLR is $>1,027$ (specificity $100 \%$; data not shown) for intrahepatic IPNB (class I), and NLR $>4.59$ or PLR $>217$ for micro-papillary IPNB (class IV).

To the best of our knowledge, this is the first study determining the utility of NLR and PLR for IPNB. There were various types of IPNB included in the study and the utility of the markers was analyzed according to the respective radiologic appearance of the tumor. Some limitations should be considered. First, because of the retrospective nature of the data, selection bias is possible. All of the patients in the current series were surgically resected, so we have no comparison with non-resected patients. Second, when we analyzed the tumors according to morphology, the sample size for some classes was quite small. Third, despite careful selection of blood samples, the level of inflammatory markers may be affected by many conditions. Finally, the nature of IPNB may vary around the world.

\section{Conclusions}

The current study provides an assessment of the utility of NLR and PLR as predictive markers for IPNB. Both markers could predict malignancy, lymph node involvement, and patient survival. PLR performed better than NLR in all categories. The markers worked very well for micro-papillary IPNB. We recommend using these markers in conjunction with the radiologic appearance of the tumors.

\section{Acknowledgements}

The authors thank Mr. Bryan Roderick Hamman for assistance with the English-language presentation of the manuscript under the aegis of the Publication Clinic KKU, Thailand.

\section{Disclosure}

The authors report no conflict of interest.

\section{References}

1. Luvira V, Pugkhem A, Bhudhisawasdi V, et al. Long-term outcome of surgical resection for intraductal papillary neoplasm of the bile duct. J Gastroenterol Hepatol 2017; 32: 527-533.

2. Luvira V, Somsap K, Pugkhem A, et al. Morphological classification of intraductal papillary neoplasm of the bile duct with survival correlation. Asian Pac J Cancer Prev 2017; 18: 207-213.

3. Dupré A, Malik HZ. Inflammation and cancer: What a surgical oncologist should know. Eur J Surg Oncol 2018; 44: 566-570.

4. Bowen RC, Little NAB, Harmer JR, et al. Neutrophil-to-lymphocyte ratio as prognostic indicator in gastrointestinal cancers: a systematic review and meta-analysis. Oncotarget 2017; 8: 32171-32189.

5. Tang H, Lu W, Li B, et al. Prognostic significance of neutrophil-to-lymphocyte ratio in biliary tract cancers: a systematic review and meta-analysis. Oncotarget 2017; 8: 36857-36868.

6. Najjar M, Agrawal S, Emond JC, et al. Pretreatment neutrophil-lymphocyte ratio: useful prognostic biomarker in hepatocellular carcinoma. J Hepatocell Carcinoma 2018; 5: 17-28.

7. Xue T-C, Zhang L, Xie X-Y, et al. Prognostic significance of the neutrophil-to-lymphocyte ratio in primary liver cancer: a metaanalysis. PLoS One 2014; 9: e96072.

8. Arima K, Okabe H, Hashimoto D, et al. The neutrophil-to-lymphocyte ratio predicts malignant potential in intraductal papillary mucinous neoplasms. J Gastrointest Surg 2015; 19: 2171-2177.

9. Gemenetzis G, Bagante F, Griffin JF, et al. Neutrophil-to-lymphocyte ratio is a predictive marker for invasive malignancy in intraductal papillary mucinous neoplasms of the pancreas. Ann Surg 2017; 266: 339-345.

10. Nakanuma Y, Harada K, Sasaki M, et al. Proposal of a new disease concept 'biliary diseases with pancreatic counterparts'. Anatomical and pathological bases. Histol Histopathol 2014; 29: 1-10.

11. Luvira V, Pugkhem A, Tipwaratorn T, et al. Simultaneous extensive intraductal papillary neoplasm of the bile duct and pancreas: a very rare entity. Case Rep Surg 2016; 2016: 1518707.

12. Gomez D, Morris-Stiff G, Toogood GJ, et al. Impact of systemic inflammation on outcome following resection for intrahepatic cholangiocarcinoma. J Surg Oncol 2008; 97: 513-518.

13. Lin G, Liu Y, Li S, et al. Elevated neutrophil-to-lymphocyte ratio is an independent poor prognostic factor in patients with intrahepatic cholangiocarcinoma. Oncotarget 2016; 7: 50963-50971.

14. Buettner S, Spolverato G, Kimbrough CW, et al. The impact of neutrophil-to-lymphocyte ratio and platelet-to-lymphocyte ratio among patients with intrahepatic cholangiocarcinoma. Surgery 2018; 164: 411-418.

15. Kitano Y, Yamashita Y-I, Yamamura K, et al. Effects of preoperative neutrophil-to-lymphocyte and platelet-to-lymphocyte ratios on survival in patients with extrahepatic cholangiocarcinoma. Anticancer Res 2017; 37: 3229-3237.

16. Hakeem AR, Marangoni G, Chapman SJ, et al. Does the extent of lymphadenectomy, number of lymph nodes, positive lymph node ratio and neutrophil-lymphocyte ratio impact surgical outcome of perihilar cholangiocarcinoma? Eur J Gastroenterol Hepatol 2014; 26: 1047-1054.

17. Wan X-S, Xu Y-Y, Qian J-Y, et al. Intraductal papillary neoplasm of the bile duct. World J Gastroenterol 2013; 19: 8595-8604.

18. Nakagawa T, Kamiyama T, Kurauchi N, et al. Number of lymph node metastases is a significant prognostic factor in intrahepatic cholangiocarcinoma. World J Surg 2005; 29: 728-733. 